\section{ASH 2017 - Proteasom-Inhibitor der zweiten Generation bewährt sich}

\section{Myelompatienten profitieren von Carfilzomib mit OS-Vorteil}

Die Integration neuer Substanzen hat die Therapie des multiplen Myeloms (MM) innerhalb kurzer Zeit stark bereichert und die Prognose betroffener Patienten verbessert. So haben sich Carfilzomib-basierte Regime in der Rezidivtherapie als sehr effektiv erwiesen. Die auf dem ASH 2017 vorgestellte Studie ASPIRE ist bereits die zweite Phase-III-Studie, in der mit einem solchen Regime eine signifikante Verlängerung des Gesamtüberlebens (OS) bei Patienten mit rezidiviertem oder refraktärem MM (rrMM) erreicht wurde.

ENDEAVOR war die erste Phase-III-Studie, in der sich das Regime mit Carfilzomib/Dexamethason im Vergleich zum Standard Bortezomib/Dexamethason durch einen signifikanten OS-Vorteil auszeichnete, erinnerte Prof. Keith Stewart, Scottsdale/USA (1). In der mit fast 800 rrMM-Patienten großen Phase-III-Studie ASPIRE hat sich das Tripelregime mit Carfilzomib, Lenalidomid, Dexamethason (KRd) auch gegenüber Lenalidomid/Dexamethason (Rd) als überlegen erwiesen: Erreicht wurden eine dreimal so hohe Rate mindestens kompletter Remissionen ( $\geq C R ; 31,6 \%$ vs. 9,3\%), eine Verlängerung des progressionsfreien Überlebens (PFS) um fast neun Monate (26,3 vs. 17,6 Monate) und eine bessere Lebensqualität $(2,3)$.

\section{OS-Reduktion um relativ 21 Prozent}

Das OS war ein sekundärer Endpunkt der ASPIRE-Studie. Dabei ergab sich für das KRdRegime ein signifikanter OS-Vorteil von knapp acht Monaten: Patienten im Kontrollarm mit Rd überlebten median 40,4 Monate, die mit dem Dreierregime behandelten dagegen 48,3 Monate. Dies entspricht einer Reduktion des Mortalitätsrisikos um relativ $21 \%$ (Hazard Ratio [HR 0,79; $p=0,0045)$. Nach 18 Monaten war bei $17,9 \%$ der Patienten unter KRd sowie bei 24,5\% derjenigen im Rd-Arm ein Ereignis aufgetreten (HR 0,69) ( $>$ Abb. 1).

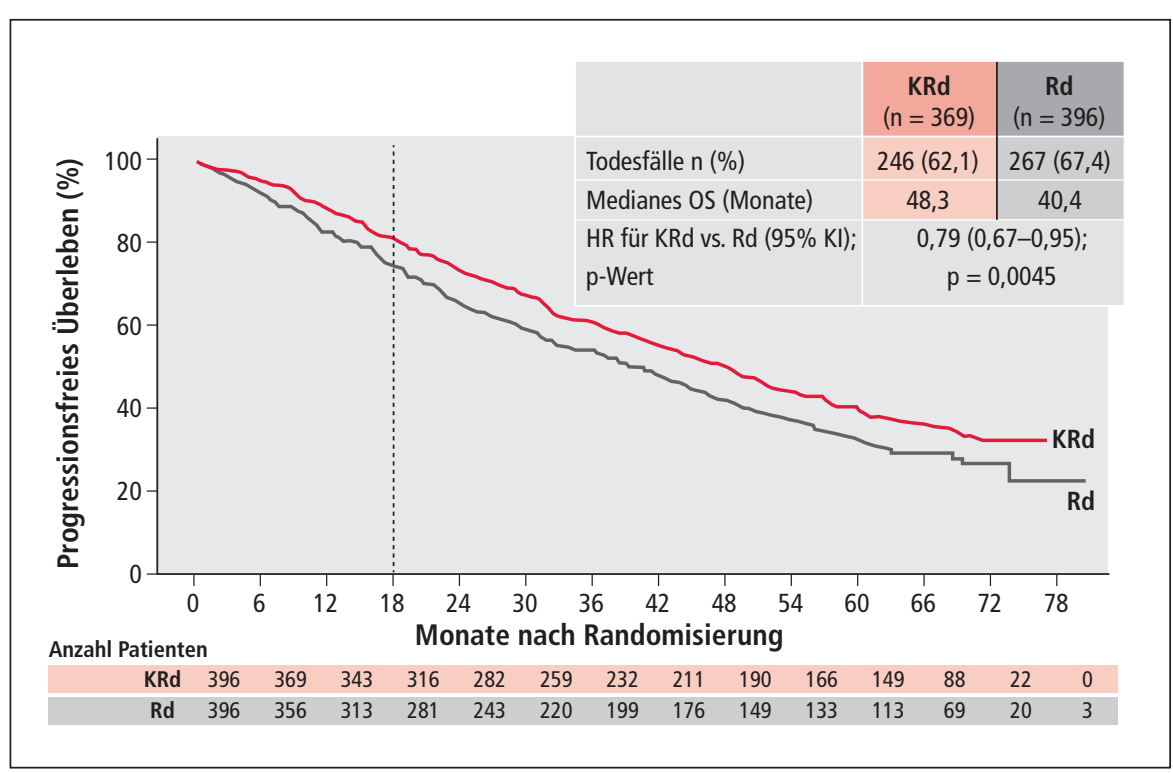

Abb. 1 ASPIRE-Studie: Signifikante Verlängerung des Gesamtüberlebens (OS) durch Carfilzomib, Lenalidomid, Dexamethason (KRd) vs. Lenalidomid/Dexamethason (Rd) (mod. nach [4])
Besonders stark profitierten laut Stewart Patienten mit nur einer Vortherapie, bei denen $\mathrm{KRd}$ das OS gegenüber dem Kontrollarm um 11,4 Monate verlängerte (47,3 vs. 35,9 Monate; HR 0,81, $95 \%$ Konfidenzintervall [KI 0,62-1,06). Patienten, die vor ihrem ersten Rückfall transplantiert worden waren, überlebten unter KRd sogar 57,2 Monate (vs. 38,6 Monate). Beim PFS zeigte sich ebenfalls ein anhaltender signifikanter Vorteil zugunsten von KRd (26,1 vs. 16,6 Monate; HR 0,66; $p<0,0001)$. Von der zusätzlichen CarfilzomibGabe profitierten auch Hochrisikopatienten mit einer PFS-Verlängerung um neun Monate.

In puncto Verträglichkeit bestätigten sich die bisherigen Daten; neue Sicherheitssignale wurden im Rahmen der erweiterten Nachbeobachtung nicht festgestellt. Therapieabbrüche erfolgten bei 19,9\% unter KRd und bei $21 \%$ unter Rd. Zwar waren Nebenwirkungen unter KRd häufiger als mit Rd. Stewart wies jedoch darauf hin, dass die Therapiedauer mit median 88 Wochen erheblich länger war als im Kontrollarm mit 57 Wochen. Nebenwirkungen Grad $\geq 3$ traten bei $87 \%$ unter KRd und $83 \%$ unter Rd auf. Darunter waren akutes Nierenversagen in 3,8\% bzw. 3,3\%, Herzversagen bei $4,3 \%$ bzw. 2,1\%, ischämische Herzerkrankungen bei 3,8\% bzw. 2,3\%, Hypertonie bei $6,4 \%$ bzw. 2,3\% und Thrombozytopenien bei $20,2 \%$ bzw. 14,9\%.

Der Onkologe bezeichnete das KRd-Regime daher zusammenfassend als exzellente Option mit belegtem OS-Vorteil für rrMM-Patienten. Einen besonders großen Benefit haben nach seinen Worten weniger stark vorbehandelte Patienten im ersten Rezidiv.

\section{Dr. Katharina Arnheim, Freiburg}

\section{Literatur}

1. Dimopoulos MA et al. Lancet Oncol 2017; 18 1327-1337.

2. Stewart AK et al. N Engl J Med 2015; 372: 142-152.

3. Stewart AK et al. J Clin Oncol 2016; 34: 3921-3930.

4. Stewart AK et al. ASH 2017; Abstract 743.

Quelle: $59^{\text {th }}$ ASH Annual Meeting, Oral Abstract Session "Myeloma: Therapy, excluding Transplantation I", Atlanta, 11. Dezember 2017. 